\title{
Sclerosing Mesenteritis Presenting as a Pseudotumor of the Greater Omentum
}

\author{
Dragan Masulovic ${ }^{a, b}$ Miodrag Jovanovic ${ }^{c} \quad$ Aleksandar Ivanovic $^{a, b}$ \\ Dejan Stojakov ${ }^{a, c}$ Marjan Micev ${ }^{d}$ Ruza Stevic ${ }^{a, b} \quad$ Aleksandar Filipovic $^{b}$ \\ Danijel Galuna, c \\ ${ }^{a}$ Faculty of Medicine, University of Belgrade, and ${ }^{b}$ Center of Radiology and MRI, ${ }^{C}$ HPB Unit and ${ }^{\mathrm{d}}$ Department of \\ Pathology, Clinic for Digestive Diseases, Clinical Center of Serbia, Belgrade, Serbia
}

\section{Key Words}

Sclerosing mesenteritis · Pseudotumor · Greater omentum

\begin{abstract}
Objective: The aim was to demonstrate a diagnostic challenge of sclerosing mesenteritis initially considered as liposarcoma. Clinical Presentation and Intervention: A 45-yearold man was admitted with a painful abdominal mass. Abdominal computed tomography demonstrated a welldemarcated tumor in his left hemiabdomen, with a large fat component and areas of soft tissue attenuation suggestive of liposarcoma. Intraoperative findings showed a tumor arising from the greater omentum. The tumor was completely removed, and histopathology confirmed a pseudotumorous type of sclerosing mesenteritis with dominant mesenteric lipodystrophy. Conclusion: This case showed that a pseudotumorous type of sclerosing mesenteritis should be considered in the differential diagnosis of the mesenteric tumors.
\end{abstract}

(c) 2015 S. Karger AG, Basel

\begin{tabular}{ll}
\hline KARGER & $\begin{array}{l}\text { (1) 2015 S. Karger AG, Basel } \\
1011-7571 / 15 / 0251-0093 \$ 39.50 / 0 \quad \text { Karger }\end{array}$ \\
E-Mail karger@karger.com & $\begin{array}{l}\text { This is an Open Access article licensed under the terms of the } \\
\text { www.karger.com/mpp }\end{array}$ \\
$\begin{array}{l}\text { Creative Commons Attribution-NonCommercial 3.0 Un- } \\
\text { ported license (CC BY-NC) (www.karger.com/OA-license), } \\
\text { applicable to the online version of the article only. Distribu- } \\
\text { tion permitted for non-commercial purposes only. }\end{array}$
\end{tabular}

\section{Introduction}

Sclerosing mesenteritis (SM) is a rare disease of unknown etiology that is characterized by a tumor-like mass composed of chronic nonspecific inflammation, fat necrosis and fibrosis $[1,2]$. The etiology is unknown, the pathogenesis is obscure and the pathological characteristics of the disease are nonspecific. Since it was first described in 1924 as 'retractile mesenteritis', a plethora of terms have been proposed, including the most frequently used mesenteric panniculitis and mesenteric lipodystrophy [1]. The rarity of this pathologic condition with protean clinical and radiological manifestations creates a diagnostic challenge $[1,3]$. Here, we report a rare case of SM initially considered as liposarcoma that affected the small intestine.

\section{Case Report}

A 45-year-old man who complained of severe abdominal pain located around the umbilicus with a palpable mass in the left upper abdominal quadrant presented to our clinic. At admission, the patient had stable vital signs, his laboratory findings were normal and he did not report any recent weight loss. Due to his abdominal discomfort and the palpable abdominal mass, an abdominal computed tomography (CT) was performed. The abdominal contrastenhanced CT demonstrated a well-demarcated $25-\mathrm{cm}$ tumor in 
Fig. 1. Contrast-enhanced abdominal CT in the axial (a) and coronal (b) plane demonstrates a well-demarcated tumor in the left hemiabdomen with a large fat component and areas of soft tissue attenuation (thin arrows) and displacement of jejunal loops (thick arrow).
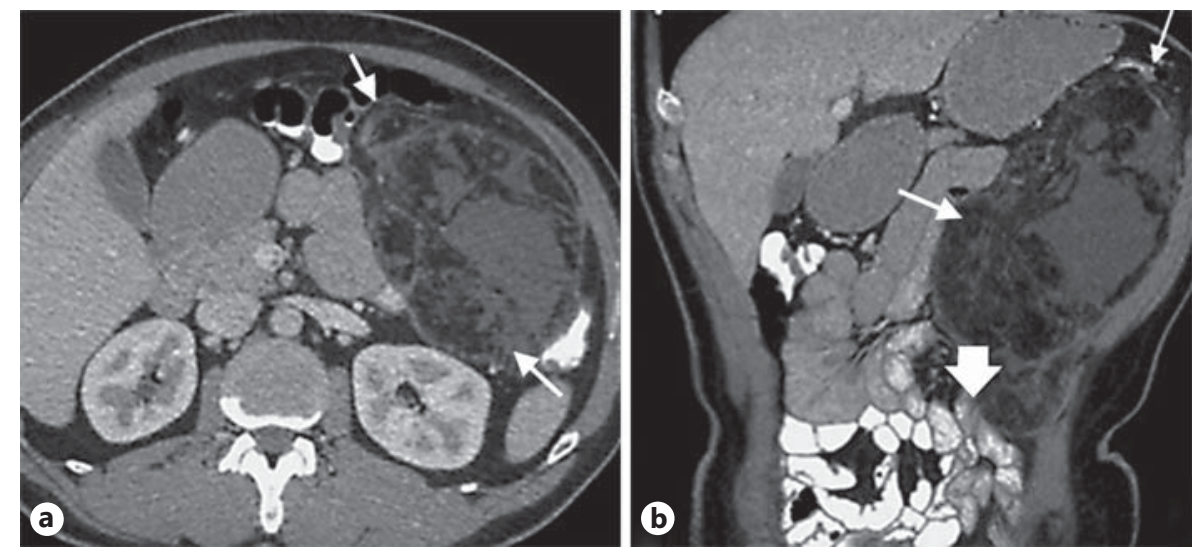

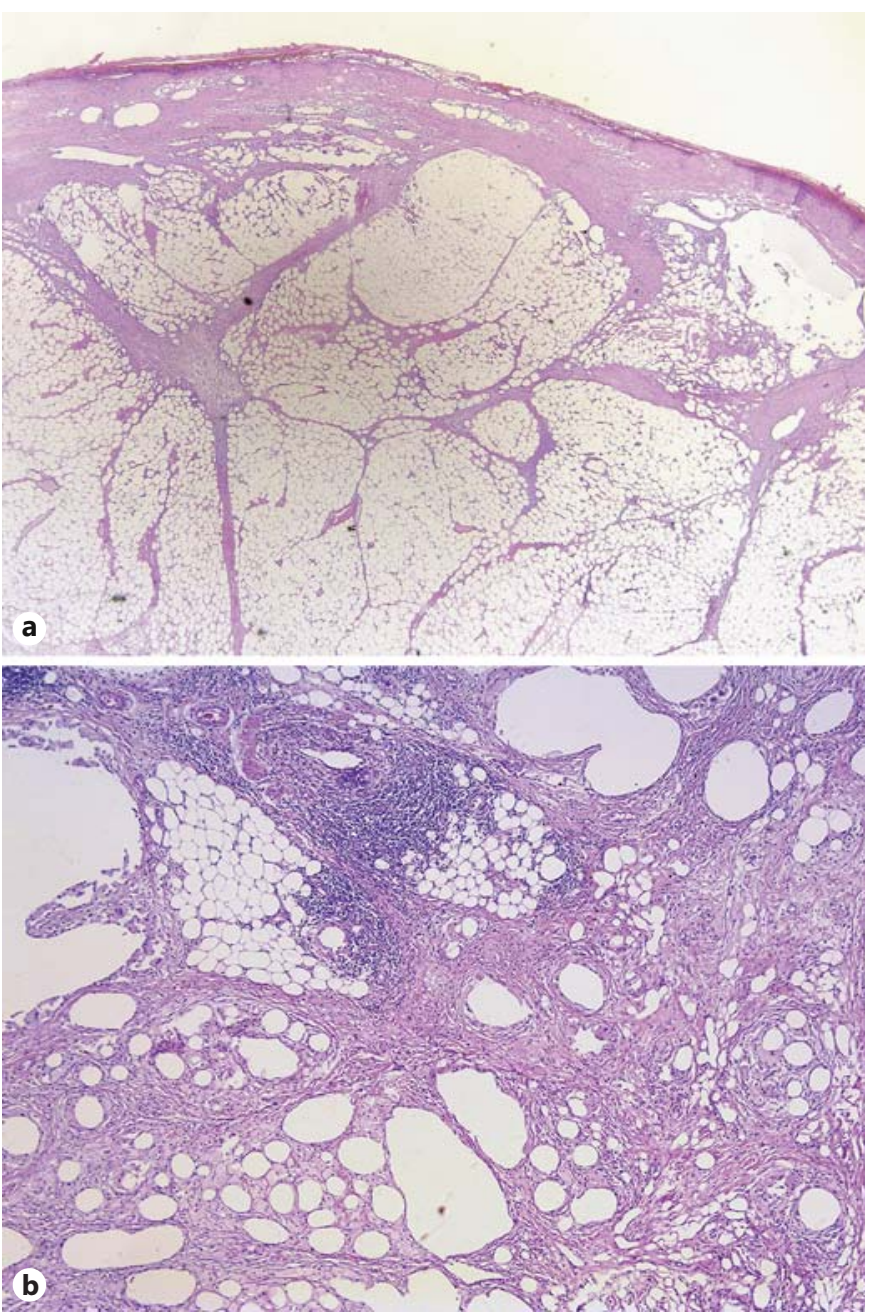

Fig. 2. Histopathological examination showed a well-defined fibrotic pseudocapsule encircling a pseudotumorous lesion (a), and variable proportions of steatonecrosis, numerous lytic microcysts and irregular surrounding fibroinflammatory reactions with abundant pools of foamy histiocytes (b). the left hemiabdomen. The tumor was encapsulated in dense fibrosis and displaced surrounding jejunal loops to the midline (fig. 1). A large fat component and areas of soft tissue attenuation in the posterior and lateral part of the tumor suggested a diagnosis of liposarcoma, although a differential diagnosis of SM was also considered. Calcification in the tumor area was absent.

Considering the tumor size, unusual site of origin and other imaging features, the multidisciplinary tumor board decided to perform a surgical exploration and tumor removal. The left half of the greater omentum was infiltrated by the tumor along with $20 \mathrm{~cm}$ of the jejunum. The partial resection of the greater omentum and resection of the affected jejunal segment was performed and the tumor was excised completely. The intraoperative frozen sections failed to discriminate between the tumor-like mesenteritis and liposarcoma or other mesenchymal tumors. The microscopic frozen sections showed mesenchymal proliferation only and the malignant potential could not be estimated. Gross examination of the specimen showed a well-circumscribed and mostly pseudoencapsulated tumor with a soft to rubbery consistency. On the cut surface there was a grey-yellowish solid mass showing irregularly septated lobular architecture of fat tissue with foci of pseudocysts partly filled with hemorrhagic fluid and small scarring areas. The paraffin sections showed extensive fat necrosis, and variably expressed fibroinflammatory reactions with clusters of mononuclear inflammatory cells and lipid-laden macrophages suggestive of mesenteric lipodystrophy (fig. 2). The final pathological diagnosis was consistent with type 2 (pseudotumor) SM with a dominant 'mesenteric lipodystrophy' type of lesion, supposed to be an early stage of the disease. The postoperative course was uneventful and no recurrence of the SM was observed 24 months following surgery.

\section{Discussion}

In the case presented here an abdominal CT demonstrated encapsulated tumor with dense fibrosis, a large fat component and areas of soft tissue attenuation without calcification suggestive of a diagnosis of liposarcoma. The exact CT appearance of SM varies depending on the predominant histomorphologic substrate, i.e. fat necrosis, in- 
flammation or fibrosis [4]. SM is usually visualized as a heterogeneous mass with a large fat component and interposed linear bands with a soft tissue density, especially where inflammation and fatty necrosis are predominant components, as was the case here. Another possibility is a homogenous mass of soft tissue density in cases of retractile SM (if fibrosis and retraction are the major components) $[2,5]$. The tumor mass usually encases the mesenteric vasculature with preservation of fat around the vessels, leading to a pathognomonic CT sign termed the 'fat ring sign'. Sabate et al. [6] described the presence of a tumor pseudocapsule in $50 \%$ of patients with mesenteric panniculitis. These pseudotumors may also have a cystic component as a result of lymphatic or venous obstruction and fat necrosis. Although radiological features are nonspecific $[5,7]$, the abdominal contrast-enhanced CT with multiplanar reconstructions plays an important role in establishing the diagnosis in proper clinical settings. However, the imaging findings of neoplastic tumor can be identical to those in SM. Laboratory findings could be helpful in the differential diagnosis of SM, mostly to exclude IgG4related autoimmune fibroinflammatory diseases. In the case presented here, the possibility of IgG4-related disease was ruled out morphologically as there were no suggestive histopathological changes such as significant lymphoplasmacytic tissue infiltration accompanied by fibrosis, obliterative phlebitis and modest tissue eosinophilia. The authors have experienced several cases of IgG4-related disease in the pancreas and biliary tract with abundant lymphoplasmacytic infiltration always being present, often with a characteristic 'storiform' pattern of fibrosis, typified by a cartwheel appearance of the arranged fibroblasts and inflammatory cells. In those cases we found a predominance of IgG4-positive plasma cells and CD4+ T lymphocytes. In our patient there were mostly histiocytic and just a few plasmacytic cells in scattered inflammatory infiltra- tion. Frozen-section histology is not helpful in distinguishing a histologic profile of lipogenic proliferative tumors, as was demonstrated in the presented patient. Giant tumorlike lesions are suspicious for malignancy; their direct compressive effect on surrounding structures can compromise vascular supply and peristaltic mobility of the intestine, requiring surgical treatment. However, only a detailed histology examination of tissue samples obtained by surgery can establish a correct diagnosis and provide appropriate treatment planning. According to the literature there is no consensus about an SM treatment algorithm. Some authors recommend observation, whereas others recommend aggressive immunosuppressive therapy with prednisone and azathioprine to prevent disease progression $[7,8]$. In symptomatic patients the treatment should be individually tailored, according to the stage of the disease and persistent symptoms. The disease progression is characterized by evolution of the three histological stages. The first stage involves the degeneration of mesenteric fat (mesenteric lipodystrophy). In the second stage, termed mesenteric panniculitis, histology findings demonstrate the domination of an inflammatory infiltrate, and the most common symptoms are fever, abdominal pain and malaise. The final stage is abundant fibrosis of the adipose tissue that contributes to tissue retraction (retractile mesenteritis). In the majority of patients there is a mixture of chronic inflammation, fat necrosis and fibrosis $[5,7,9]$.

\section{Conclusion}

This case report shows that the pseudotumorous type of SM should be considered in the differential diagnosis of tumors located in the mesentery. An abdominal CT can help with the initial diagnosis, but surgical biopsy is mandatory to confirm this diagnosis.

\section{References}

$>1$ Akram S, Pardi DS, Schaffner JA, et al: Sclerosing mesenteritis: clinical features, treatment, and outcome in ninety-two patients. Clin Gastroenterol Hepatol 2007;5:589-596.

-2 Gu GL, Wang SL, Wei XM, et al: Sclerosing mesenteritis as a rare cause of abdominal pain and intraabdominal mass: a cases report. Cases J 2008; 1:242.

$>3$ Lawler LP, McCarthy DM, Fishman EK, et al: Sclerosing mesenteritis: depiction by multidetector CT and three-dimensional volume rendering. Am J Roentgenol 2002;178:97-99.
4 Vlachos K, Archontovasilis F, Falidas E: Sclerosing mesenteritis: diverse clinical presentations and dissimilar treatment options: a case series and review of the literature. Int Arch Med 2011;4:17.

5 Issa I, Baydoun H: Mesenteric panniculitis: various presentations and treatment regimens. World J Gastroenterol 2009;15:38273830 .

6 Sabate JM, Torrubia S, Maideu J, et al: Sclerosing mesenteritis: imaging findings in $17 \mathrm{pa}$ tients. Am J Roentgenol 1999;172:625-629.
7 Ali FN, Ishaque S, Jamil B, et al: Sclerosing mesenteritis as a cause of abdominal mass and discomfort in an elderly patient: a case report and literature review. Case Rep Med 2010; 2010:625321.

-8 Daskalogiannaki M, Voloudaki A, Prassopoulos P, et al: CT evaluation of mesenteric panniculitis: prevalence and associated diseases. Am J Roentgenol 2000;174:427-431.

$\checkmark 9$ Vlachos K, Archontovasilis F, Falidas E: Sclerosing mesenteritis: diverse clinical presentations and dissimilar treatment options: a case series and review of the literature. Int Arch Med 2011;4:17. 